
\title{
S Research S guare ons
}

\section{Simultaneous Monitoring of the Middle Cerebral and Basilar Arteries to Detect Right-to-Left Shunts Using Transcranial Doppler by Agitated Saline Administration}

\section{Min Kim}

Ajou University School of Medicine

So Young Park

Ajou University School of Medicine

Ji Man Hong ( $\nabla$ dacda@hanmail.net)

Ajou University School of Medicine

\section{Research Article}

Keywords: transcranial doppler, middle cerebral artery, basilar artery, right to left shunt

Posted Date: October 14th, 2021

DOl: https://doi.org/10.21203/rs.3.rs-956948/v1

License: (c) (1) This work is licensed under a Creative Commons Attribution 4.0 International License.

Read Full License

Version of Record: A version of this preprint was published at Scientific Reports on April 22nd, 2022. See the published version at https://doi.org/10.1038/s41598-022-10645-7. 


\section{Abstract}

Transcranial Doppler (TCD) is an easy, non-invasive, and real-time monitoring device for detecting rightto-left shunts (RLS). Nonetheless, it has limited benefits in patients with poor temporal windows.

Therefore, we aimed to investigate whether the basilar artery (BA) window was as effective as the middle cerebral artery (MCA) in detecting RLS during TCD monitoring. Overall, we enrolled 344 patients with stroke, transient ischemic attack, headache, or dizziness. MCA and BA were monitored using a modified headset. To investigate the feasibility of the suboccipital window in detecting RLS, we instituted an evaluation tool with three tiers to evaluate microembolic signals (MESs) during TCD monitoring. Tier 1: TCD monitoring of the MCA (bilaterally) in the resting state, tier 2: TCD monitoring of the MCA (bilaterally) while performing the Valsalva maneuver, and tier 3: TCD monitoring of the index MCA and BA while performing the Valsalva maneuver. In tiers 2 and 3 , a high agreement rate of 0.8076 and 0.8068 $(p<0.001)$, respectively, on the weighted kappa index, and a high intra-class correlation coefficient of 0.9822 and $0.9860(p<0.001)$, respectively, were observed on detecting MESs. Our data suggests that the BA window is as effective as the MCA window for detecting RLS on TCD.

\section{Introduction}

Ischemic stroke without risk factors for cerebral vascular disease should be evaluated for alternative causes. ${ }^{1}$ The causes of $10-40 \%$ of all strokes are not identified despite comprehensive diagnostic testing. ${ }^{2}$ These strokes are known as cryptogenic strokes. The most common cause of a cryptogenic stroke is probably a paradoxical embolism due to a patent foramen ovale (PFO) or an atrial septal aneurysm (ASA). ${ }^{3}$ Right-to-left shunts (RLS) through PFOs or ASAs cause paradoxical embolisms, which originate in the systemic venous circulation and enter the systemic arterial circulation. Recent studies have shown that PFO closure reduces the recurrence rate of cryptogenic stroke. ${ }^{4-7}$ Therefore, it is essential to detect the presence of RLS through PFOs in cryptogenic stroke.

Transesophageal echocardiography (TEE) is a gold standard modality for detecting cardiac RLS. ${ }^{8-10}$ However, the use of TEE may be limited in patients with acute stroke who have acute illness, mental status deteriorates, and coagulopathy/bleeding tendency. Transcranial Doppler (TCD) is an easy, noninvasive, and real-time monitoring apparatus for detecting RLS. ${ }^{11,12}$ Additionally, this technique is highly sensitive, specific, and convenient for screening compared to TEE regardless of location. ${ }^{13,14}$ However, a limitation of TCD is that $10-20 \%$ of all patients cannot be evaluated because of a poor temporal window (PTW). ${ }^{15}$ Patients with a PTW account for $29-34 \%$ of the Asian population, which is a much higher incidence than that of the Caucasian population. ${ }^{16-18}$ Interestingly, in patients with provoked RLS, the ischemic lesion was found to be located predominantly in the cerebral posterior circulation system. ${ }^{19}$ Several studies have attempted to utilize the suboccipital window for detecting RLS on TCD in patients with PTWs. ${ }^{20-22}$ Therefore, the purpose of this study was to investigate if simultaneous monitoring of the basilar artery (BA) was as effective as monitoring the MCA for detecting RLS on TCD. 


\section{Results}

\section{Demographic characteristics}

The average age of the patients was $58.2 \pm 15.8$ years, and the male: female ratio was $165: 179$. Among the patients, 129 (37.5\%) had hypertension, $70(20.3 \%)$ had diabetes mellitus, and $92(26.7 \%)$ had dyslipidemia. The reasons for the test were stroke $(n=229)$, headache $(n=53), \operatorname{TIA}(n=22)$, WMHI $(n=20)$, TGA $(n=11)$, and dizziness $(n=9)$. Among these patients, $53(15.4 \%)$ had PTWs; 41 had PTWs on both sides and 12 had it on one side only (eight on the right side and four on the left side) detail the demographic characteristics of the patients in this study are shown in Table 1. 
Table 1

Demographics of enrolled patients.

\begin{tabular}{|c|c|c|c|}
\hline \multicolumn{2}{|l|}{ Demographic characteristics } & \multicolumn{2}{|c|}{ Patients ( $n=344)$} \\
\hline \multicolumn{2}{|l|}{ Age, y } & \multicolumn{2}{|c|}{$58.2 \pm 15.8$ (13 to 89$)$} \\
\hline \multicolumn{2}{|l|}{ Sex (Male/female) } & \multicolumn{2}{|l|}{$165 / 179$} \\
\hline Poor temporal window, n (\%) & & \multicolumn{2}{|c|}{$53(15.4)^{\star}$} \\
\hline \multicolumn{4}{|l|}{ Underlying disease } \\
\hline \multicolumn{2}{|l|}{ Hypertension, n (\%) } & \multicolumn{2}{|c|}{$129(37.5)$} \\
\hline \multicolumn{2}{|l|}{ Diabetes mellitus, n (\%) } & \multicolumn{2}{|l|}{$70(20.3)$} \\
\hline \multicolumn{2}{|l|}{ Dyslipidemia, n (\%) } & \multicolumn{2}{|l|}{$92(26.7)$} \\
\hline \multicolumn{2}{|l|}{ History of stroke, n (\%) } & \multicolumn{2}{|c|}{$242(70.3)$} \\
\hline \multicolumn{2}{|l|}{ Cardiac problem, n (\%) } & \multicolumn{2}{|l|}{$7(2.0)$} \\
\hline \multicolumn{4}{|c|}{ Reasons for testing using the TCD } \\
\hline \multicolumn{2}{|l|}{ Stroke, n (\%) } & \multicolumn{2}{|c|}{$229(66.6)$} \\
\hline \multicolumn{2}{|l|}{ Headache, n (\%) } & \multicolumn{2}{|l|}{$53(15.4)$} \\
\hline \multicolumn{2}{|l|}{ TIA, n (\%) } & \multicolumn{2}{|l|}{$22(6.4)$} \\
\hline \multicolumn{2}{|l|}{ WMHI, n (\%) } & \multicolumn{2}{|l|}{$20(5.8)$} \\
\hline \multicolumn{2}{|l|}{ TGA, n (\%) } & \multicolumn{2}{|l|}{$11(3.2)$} \\
\hline \multicolumn{2}{|l|}{ Dizziness, n (\%) } & \multicolumn{2}{|l|}{$9(2.6)$} \\
\hline Baseline laboratory findings & Stroke $(n=229)$ & Non-stroke $(n=115)$ & P-value \\
\hline Hemoglobin & $13.9 \pm 7.0$ & $14.0 \pm 1.31$ & 0.9053 \\
\hline Platelet & $239.2 \pm 75.0$ & $231.66 \pm 48.7$ & 0.2866 \\
\hline $\mathrm{HbA1c}$ & $6.0 \pm 1.1$ & $5.8 \pm 1.3$ & 0.2136 \\
\hline Total cholesterol & $173.0 \pm 149.8$ & $181.5 \pm 40.9$ & 0.5785 \\
\hline CRP & $0.6 \pm 1.7$ & $0.6 \pm 2.1$ & 0.8442 \\
\hline $\begin{array}{l}\text { TIA: Transient ischemic attacl } \\
\text { HbA1c: Hemoglobin A1c, CRP }\end{array}$ & $\begin{array}{l}\text { MHI: white matt } \\
\text { reactive protein }\end{array}$ & yperintensities, TGA: 1 & nsient glo \\
\hline
\end{tabular}

Outcomes of the TCD monitoring parameters in both of the MCAs while performing the Valsalva maneuver in Tier 2 
When measured simultaneously in both of the MCAs, the average number of MES were $14.97 \pm 31.18$ (range: $0-298$ ) in the right MCA, and $14.94 \pm 32.41$ (range, 0-303) in the left MCA in tier 2. The right MCA was categorized according to the International Consensus Criteria as: Grade $0(n=70)$, Grade $1(n=160)$, Grade $2(n=111)$, and Grade $3(n=3)$. In contrast, the left MCA was classified as Grade $0(n=68)$, Grade 1 $(n=167)$, Grade $2(n=106)$, and Grade $3(n=3)$ (Table 2). In addition, the right MCA was categorized based on the SLS as: Grade $0(n=70)$, Grade $\otimes(n=160)$, Grade $\otimes(n=68)$, Grade $\otimes(n=38)$, Grade $\otimes(n=7)$, and Grade $\otimes(n=1)$. In comparison, the left MCA was scored as Grade $0(n=68)$, Grade $\otimes(n=167)$, Grade $\otimes(n=65)$, Grade $\otimes(n=37)$, Grade $\otimes(n=7)$, and Grade $\otimes(n=0)$.

Table 2

Categorization as per the International Consensus Criteria in tiers 2 and 3

\begin{tabular}{|c|c|c|c|c|c|c|c|}
\hline \multirow{2}{*}{\multicolumn{2}{|c|}{$\begin{array}{l}\text { Tier } 2 \\
\text { (R-MCA vs L-MCA) }\end{array}$}} & \multicolumn{4}{|l|}{ Left (L) } & \multirow{3}{*}{$\begin{array}{l}\text { Total } \\
70\end{array}$} & \multirow[t]{3}{*}{ P-value } \\
\hline & & \multirow{2}{*}{$\begin{array}{l}\text { Grade } 0 \\
22\end{array}$} & \multirow{2}{*}{$\begin{array}{l}\text { Grade } 1 \\
48\end{array}$} & \multirow{2}{*}{$\begin{array}{l}\text { Grade } 2 \\
0\end{array}$} & \multirow{2}{*}{$\begin{array}{l}\text { Grade } 3 \\
0\end{array}$} & & \\
\hline Right (R) & Grade 0 & & & & & & \\
\hline & Grade 1 & 46 & 106 & 8 & 0 & 160 & \\
\hline & Grade 2 & 0 & 13 & 98 & 0 & 111 & \\
\hline & Grade 3 & 0 & 0 & 0 & 3 & 3 & \\
\hline & Total & 68 & 167 & 106 & 3 & 344 & \\
\hline \multicolumn{2}{|c|}{ Weighted Kappa index } & & & & & 0.6866 & $p<0.001$ \\
\hline \multirow{2}{*}{\multicolumn{2}{|c|}{$\begin{array}{l}\text { Tier } 3 \\
\text { (iMCA vs BA) }\end{array}$}} & \multicolumn{4}{|l|}{ BA } & Total & P-value \\
\hline & & Grade 0 & Grade 1 & Grade 2 & Grade 3 & & \\
\hline \multirow[t]{5}{*}{ iMCA } & Grade 0 & 78 & 18 & 0 & 0 & 96 & \\
\hline & Grade 1 & 17 & 104 & 1 & 0 & 122 & \\
\hline & Grade 2 & 0 & 2 & 78 & 0 & 80 & \\
\hline & Grade 3 & 0 & 0 & 0 & 5 & 5 & \\
\hline & Total & 95 & 124 & 79 & 5 & 303 & \\
\hline \multicolumn{6}{|c|}{ Weighted Kappa index } & 0.8119 & $p<0.001$ \\
\hline
\end{tabular}

The weighed kappa index was $0.6866(p<0.001)$ in both the MCAs when assessed using the International Consensus Criteria. In addition, the weighed kappa index was $0.8076(p<0.001)$ when both the MCAs were evaluated using the SLS. The ICC was $0.9822(p<0.001)$ on comparing the number of MESs measured in both MCAs (Fig. 3). 
Outcomes of the TCD monitoring parameters in both the index MCA and BA while performing the Valsalva maneuver in Tier 3

The iMCA and BA were measured in 303 patients, after excluding 41 out of 344 patients due to PTWs on both sides. Out of a total of 303 patients, $182(60.1 \%)$ on the right and $121(39.9 \%)$ on the left MCA were measured simultaneously with the BA. When MES was measured simultaneously, the average number of bubbles was $14.15 \pm 36.32$ (range: $0-505$ ) in the iMCA and $14.35 \pm 38.85$ (range: $0-559$ ) in the BA. The MCA was categorized using the International Consensus Criteria as: Grade $0(n=96)$, Grade $1(n=122)$, Grade $2(n=80)$, and Grade $3(n=5)$. The BA was grouped as: Grade $0(n=95)$, Grade $1(n=124)$, Grade 2 $(n=79)$, and Grade $3(n=5)$ (Table 2). The weighted kappa index was $0.8119(p<0.001)$ when estimated

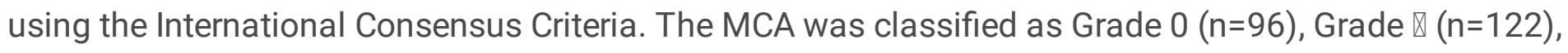
Grade $\otimes(n=42)$, Grade $\otimes(n=39)$, Grade $\otimes(n=3)$, and Grade $\otimes(n=1)$; and the $B A$ as Grade $0(n=95)$, Grade $\otimes$ $(n=124)$, Grade $\otimes(n=42)$, Grade $\otimes(n=38)$, Grade $\otimes(n=3)$, and Grade $\otimes(n=1)$ (Table 3$)$. The weighted kappa index was $0.8069(p<0.001)$ when evaluated using the SLS. The measurements for both the MCA and BA were similar when compared with the MCA measurements bilaterally. The ICC was $0.9860(p<0.001)$ on comparing the number of bubbles measured in the iMCA and BA (Fig. 3). 
Table 3

Categorization as per the Spencer logarithmic scale in tiers 2 and 3

\begin{tabular}{|c|c|c|c|c|c|c|c|c|c|}
\hline \multirow{2}{*}{\multicolumn{2}{|c|}{$\begin{array}{l}\text { Tier } 2 \\
\text { (L-MCA vs R-MCA) }\end{array}$}} & \multicolumn{6}{|c|}{ Left (L) } & \multirow[t]{2}{*}{ Total } & \multirow[t]{2}{*}{ P-value } \\
\hline & & 0 & [ & प & [ & [ & प & & \\
\hline & 0 & 22 & 48 & 0 & 0 & 0 & 0 & 70 & \\
\hline \multirow[t]{7}{*}{ Right (R) } & $\square$ & 46 & 106 & 8 & 0 & 0 & 0 & 160 & \\
\hline & प & 0 & 13 & 53 & 2 & 0 & 0 & 68 & \\
\hline & प & 0 & 0 & 4 & 32 & 2 & 0 & 38 & \\
\hline & प & 0 & 0 & 0 & 3 & 4 & 0 & 7 & \\
\hline & प & 0 & 0 & 0 & 0 & 1 & 0 & 1 & \\
\hline & Total & 68 & 167 & 65 & 37 & 7 & 0 & 344 & \\
\hline & & \multicolumn{6}{|c|}{ Weighted Kappa index } & 0.8076 & $p<0.001$ \\
\hline \multirow{2}{*}{\multicolumn{2}{|c|}{$\begin{array}{l}\text { Tier } 3 \\
\text { (iMCA vs BA) }\end{array}$}} & \multicolumn{6}{|c|}{ BA } & Total & P-value \\
\hline & & 0 & प & प & प & 0 & प & & \\
\hline \multirow[t]{7}{*}{ iMCA } & 0 & 78 & 18 & 0 & 0 & 0 & 0 & 96 & \\
\hline & प & 17 & 104 & 1 & 0 & 0 & 0 & 122 & \\
\hline & प & 0 & 2 & 39 & 1 & 0 & 0 & 42 & \\
\hline & प & 0 & 0 & 2 & 37 & 0 & 0 & 39 & \\
\hline & प & 0 & 0 & 0 & 0 & 3 & 0 & 3 & \\
\hline & प & 0 & 0 & 0 & 0 & 0 & 1 & 1 & \\
\hline & Total & 95 & 124 & 42 & 38 & 3 & 1 & 303 & \\
\hline \multicolumn{8}{|c|}{ Weighted Kappa index } & 0.8068 & $p<0.001$ \\
\hline
\end{tabular}

\section{Discussion}

This study shows that the suboccipital window for insonation of the BA is as effective as the temporal window for MCA in detecting RLS using the TCD.

Approximately $10-20 \%$ of stroke patients have PTWs that prevent sufficient TCD insonation of cerebral vessels around the circle of Willis. The PTW rates are higher in Asians than in Caucasians. ${ }^{14,16}$ It was difficult to evaluate RLS using TCD in patients with PTWs. Therefore, some studies had attempted to 
detect RLS using the suboccipital windows, submandibular ICA, or the orbital windows rather than the temporal windows. ${ }^{20-23}$

One study identified MESs using the orbital window for ICA and the temporal window for MCA to detect RLS, and not the suboccipital window in a TCD study. ${ }^{23}$ Another study compared the monitoring of cervical arteries (submandibular ICA and vertebral artery [VA]) to that of the MCA. The study addressed that the TCD of the cervical submandibular ICA and VA is a valid screening method to detect RLS due to a PFO. ${ }^{21}$

Most studies have replaced the temporal window due to a PTW with a suboccipital window to detect RLS using the TCD. The studies had demonstrated the usefulness of the suboccipital window (for monitoring the VA) in detecting RLS using the TCD. ${ }^{21,22}$ Although there studies had determined that the suboccipital window had a diagnostic ability that was equal to that of the MCA, there are no quantitative studies detecting the extent of RLS, or comparing the temporal and suboccipital windows using correlation coefficients and reliable statistics. Additionally, several studies have shown that ischemic lesions are more common in the posterior cerebral circulation of patients with RLS found using the Valsalva maneuver. ${ }^{19}$ Therefore, the suboccipital window might be a better alternative than the submandibular ICA or orbital window in patients with PTWs.

A quantitative study conducted by Guo et al. that compared the left MCA and the left VA for the detection of RLS showed no significant differences between the two (including constant and provoked RLS). Besides, the sensitivity and specificity of monitoring (using the left VA) were relatively higher with the left MCA upheld as the standard. ${ }^{22}$ However, that study had also several limitations. First, the probe for VA monitoring was manually positioned. The VA might have been missed while testing if its course had changed. The VA has many variations including hypoplasia. ${ }^{24,25}$ As it is difficult to monitor the VA using the TCD under the same condition, it can be difficult to detect RLS. Second, TCD monitoring was performed in the right lateral decubitus position, although this was not the most optimal position for detecting RLS. ${ }^{26,27}$

Therefore, we chose the BA instead of VA and compared it to the MCA in our study. The advantages of using agitated saline while performing the TCD in a suboccipital window (for insonation of the BA) can be explained by several reasons. First, the VA is not affected due to PTWs, but is occasionally hypoplastic. VA hypoplasia (VAH) is not an uncommon congenital variation of the VAs leading to asymmetry. VAH with caliber discrepancies of more than $1: 1.7$ was observed in up to $10 \%$ of normal individuals. ${ }^{24,25}$ Second, the amount of blood in the vertebrobasilar system is lower than that (about $20 \%$ ) in the carotid circulation. ${ }^{28} \mathrm{~A}$ study had evaluated the flow in the proximal and distal cerebral arteries using high-resolution phase-contrast magnetic resonance imaging. The study showed that the total cerebral blood flow $(717 \pm 123 \mathrm{~mL} / \mathrm{min}$ ) was distributed (on each side) as follows: middle cerebral artery, $21 \%$; distal MCA, $6 \%$; anterior cerebral artery (ACA), $12 \%$; distal ACA, $4 \%$; ophthalmic artery, $2 \%$; posterior cerebral artery, $8 \%$; and basilar artery, $20 \% .{ }^{29}$ The BA has a similar amount of blood flow as that in the 
MCA. Third, the headset could be fixed to the BA during TCD monitoring. Therefore, it was much easier to use as the sonographer did not have to fix the probe manually during TCD study in the suboccipital window. This also enabled the study of patients in various position, such as the prone and sitting positions. Some studies have shown that the upright sitting position is the best position for detecting RLS. ${ }^{26,27}$ This was relatively more comfortable and easily achieved.

Previous randomized controlled trials did not reveal any statistical significance between PFO closure and medical therapy for reducing stroke rates. ${ }^{30-32}$ However, some recent studies have suggested that PFO closure is more effective than medical therapy for reducing recurrent stroke rates. ${ }^{4-7}$ The benefits observed in recent trials following PFO closure is probably related to several factors involved in the selection of patients with PFO features, such as a large shunt size, those with a more severe presentation or presence of an atrial septal aneurysm. ${ }^{4,6,7}$ Therefore, it is important to evaluate the size and the severity of the PFO with RLS. TCDs could be an alternative to TEE for assessing the severity of PFO and a useful follow-up test for patients after PFO closure. We believed that TCD monitoring for BA can also be a method for quantitatively assessing the severity of PFO.

Our study has several limitations. First, the MCA and BA were compared only in provoked RLS. A comparative study of the MCA and BA is needed in constant RLS. Second, this study has not proven that ischemic lesions are located predominantly in the vertebrobasilar circulation, especially in patients with

provoked RLS. ${ }^{19}$ A previous study showed that the time taken for appearance of the bubble after administering the injection was higher in the vertebrobasilar circulation. ${ }^{20}$ In future studies, the number of bubbles in addition to the time of occurrence of the bubbles needs to be considered while comparing the MCA and BA. Third, the BA may also present with hypoplasia and have lower blood flow than that in the carotid circulation. This may be associated with bilateral VAH or bilateral fetal-type posterior circle of Willis. We identified 21 patients with BA hypoplasia (diameter $<2 \mathrm{~mm}$ ) in our study. Even with the basilar artery hypoplasia, the weighted kappa index was 0.8956 when categorized using the SLS in Tier 3 . Additionally, there were no patients in whom the BA was not detected using the TCD.

\section{Methods}

\section{Participants}

This retrospective study was reviewed and approved by the Institutional Review Board (IRB) of Ajou University Hospital, Suwon, Korea (AJIRB-MED-MDB-21-349).The Declaration of Helsinki, was followed. The requirement for informed consent was waived by IRB of Ajou University Hospital owing to the retrospective nature of this study.

Patients tested using agitated saline TCD from December, 2015 to September, 2020 were recruited in this retrospective study. A total of 816 patients with headaches, transient global amnesia (TGA), white matter hyperintensities (WMHI), transient ischemic attack (TIA), or stroke were tested using the TCD at Ajou University Hospital. The study excluded patients who did not reveal any microembolic signals (MESs) on 
TCD while in the resting state or on performing the Valsalva maneuver $(n=472)$. Therefore, a total of 344 patients were included in the study. Figure 1 shows a detailed flowchart for the recruitment of this study population.

\section{The TCD procedure}

The tests were performed in the following manner. An evaluation tool with three tiers was instituted to evaluate MESs during TCD monitoring. In tier 1, the TCD test was performed in both the right and left MCAs (or internal carotid arteries [ICAs] with a PTW) in the resting state. In tier 2, the TCD test was carried out after administering agitated saline for $>3$ min while performing the Valsalva maneuver. In tier 3 , if one or more MESs were detected from tier 1 or 2, MCA on one side (termed as the index MCA [iMCA]) was tested along with BA for $>3$ min after performing the Valsalva maneuver. However, patients with bilateral PTWs were excluded 41 in tier 3 (Fig. 1).

The patients were asked to exhale into a manometer and maintain a pressure of $40 \mathrm{mmHg}$ for $10 \mathrm{~s}$ to trigger the Valsalva maneuver. The iMCA was defined as MCA on one side, either right or left that had better visibility or detectability for MES. Microbubbles were provoked by administering $9 \mathrm{~mL}$ of $0.9 \%$ saline mixed with $1 \mathrm{~mL}$ of the patient's blood and $1 \mathrm{~mL}$ of air. All 344 patients underwent TCD examinations as described in tiers 1 and 2. TCD as defined in tier 3, was performed in a total of 303 patients after excluding those with bilateral PTWs $(n=41)$.

A TCD examination was performed by sonographers using the PMD 150 M (Spencer Technologies, Seattle, WA) or TC8080 (Pioneer TC 8080; Viasys Healthcare, Madison, WI) with a 2 MHz probe. MES were recorded using probes mounted on the modified headset (Phifix; Astron, Suwon, Republic of Korea) that was monitored for both MCA and BA at the same time in detecting RLS using TCD (Fig. 2). There are two probes on the headset. One probe is placed on the temporal window to detect the MCA and the other probe is placed on the suboccipital window to detect the BA (Fig. 2). When the iMCA and BA were monitored simultaneously for MES using TCD, the patients were instructed to wear the headset and perform the Valsalva maneuver. MES was defined as visible (short duration, high-intensity and unidirectional) and audible (click, chirp, or whistles) signals in the Doppler flow spectrum. All the MES were reviewed by two neurologists and sonographers of more than 10 years' experience.

\section{MES grading}

We used two grading systems for categorizing the MESs: the International Consensus Criteria and Spencer's Logarithmic Scale (SLS). ${ }^{33,34}$ The patients were divided into four groups based on the MES using the International Consensus Criteria as follows: Grade 0, no occurrence of microbubbles; Grade 1, 1-10 microbubbles; Grade 2, >10 microbubbles and shower pattern; Grade 3, curtain pattern. ${ }^{33}$ The MESs were also categorized into six groups based on the SLS: Grade 0, no occurrence of microbubbles; Grade $\nabla$,

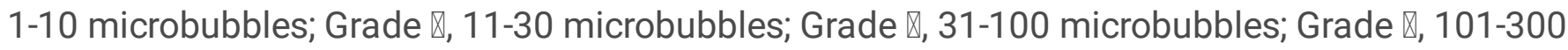

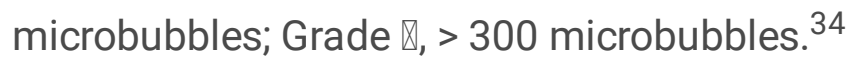




\section{Statistical analysis}

Statistical analyses were performed using the R Statistical Software (version 3.6.3.; R Foundation for Statistical Computing, Vienna, Austria). To verify the consistency of the agreement tests in various tiers, the analysis was performed in two ways. First, the weighted kappa index was used to compare the agreement between the right and left MCA, and the agreement between the iMCA and BA after classification based on the International Consensus Criteria and SLS in tiers 2 and 3. Second, MES identified quantitatively in the left and right MCA were verified and compared using the intraclass correlation coefficient (ICC) in tier 2. Similarly, the microbubbles identified in the iMCA and BA were verified, and compared using the ICC in tier 3 . $P<0.05$ was considered statistically significant.

\section{Declarations}

\section{Acknowledgements}

None

\section{Conflict of interests}

The authors have no relevant conflicts of interest to disclose.

\section{Role of the funding source:}

None

\section{References}

1 Kizer, J. R. \& Devereux, R. B. Patent foramen ovale in young adults with unexplained stroke. New England Journal of Medicine353, 2361-2372 (2005).

2 Saver, J. L. Cryptogenic stroke. New England Journal of Medicine374, 2065-2074 (2016).

3 Messe, S. R. \& Kasner, S. E. Patent foramen ovale in cryptogenic stroke: not to close. Circulation118, 1999-2004 (2008).

4 Mas, J.-L. et al. Patent foramen ovale closure or anticoagulation vs. antiplatelets after stroke. New England Journal of Medicine377, 1011-1021 (2017).

5 Saver, J. L. et al. Long-term outcomes of patent foramen ovale closure or medical therapy after stroke. New England Journal of Medicine377, 1022-1032 (2017).

6 Søndergaard, L. et al. Patent foramen ovale closure or antiplatelet therapy for cryptogenic stroke. New England Journal of Medicine377, 1033-1042 (2017). 
7 Lee, P. H. et al. Cryptogenic stroke and high-risk patent foramen ovale: the DEFENSE-PFO trial. Journal of the American College of Cardiology71, 2335-2342 (2018).

8 Pinto, F. When and how to diagnose patent foramen ovale. Heart91, 438-440 (2005).

9 Pearson, A. C., Labovitz, A. J., Tatineni, S. \& Gomez, C. R. Superiority of transesophageal echocardiography in detecting cardiac source of embolism in patients with cerebral ischemia of uncertain etiology. Journal of the American College of Cardiology17, 66-72 (1991).

10 Job, F. P. et al. Comparison of transcranial contrast Doppler sonography and transesophageal contrast echocardiography for the detection of patent foramen ovale in young stroke patients. The American journal of cardiology74, 381-384 (1994).

$11 \mathrm{Nemec}$, J. J. et al. Comparison of transcranial Doppler ultrasound and transesophageal contrast echocardiography in the detection of interatrial right-to-left shunts. The American journal of cardiology68, 1498-1502 (1991).

$12 \mathrm{Di}$ Tullio, M. et al. Comparison of diagnostic techniques for the detection of a patent foramen ovale in stroke patients. Stroke24, 1020-1024 (1993).

13 Jauss, M., Kaps, M., Keberle, M., Haberbosch, W. \& Dorndorf, W. A comparison of transesophageal echocardiography and transcranial Doppler sonography with contrast medium for detection of patent foramen ovale. Stroke25, 1265-1267 (1994).

14 Van, H. et al. Sensitivity of transcranial Doppler versus intracardiac echocardiography in the detection of right-to-left shunt. JACC: Cardiovascular Imaging3, 343-348 (2010).

15 Seidel, G., Kaps, M. \& Gerriets, T. Potential and limitations of transcranial color-coded sonography in stroke patients. Stroke26, 2061-2066 (1995).

16 Itoh, T. et al. Rate of successful recording of blood flow signals in the middle cerebral artery using transcranial Doppler sonography. Stroke24, 1192-1195 (1993).

17 Lien, L. M. et al. Comparison of transcranial color-coded sonography and magnetic resonance angiography in acute ischemic stroke. Journal of Neuroimaging11, 363-368 (2001).

18 Kwon, J. H., Kim, J. S., Kang, D. W., Bae, K. S. \& Kwon, S. U. The thickness and texture of temporal bone in brain CT predict acoustic window failure of transcranial Doppler. Journal of Neuroimaging16, 347-352 (2006).

19 Kim, B. J., Kim, N.-Y., Kang, D.-W., Kim, J. S. \& Kwon, S. U. Provoked right-to-left shunt in patent foramen ovale associates with ischemic stroke in posterior circulation. Stroke45, 3707-3710 (2014). 
20 Del Sette, M. et al. Diagnosis of right-to-left shunt with transcranial Doppler and vertebrobasilar recording. Stroke38, 2254-2256 (2007).

21 Perren, F., Kremer, C., Iwanovski, P., Savva, E. \& Landis, T. Detection of Right-to-Left Cardiac Shunt in the Absence of Transcranial Acoustic Bone. Journal of Neuroimaging26, 269-272 (2016).

22 Guo, Y.-Z. et al. Comparison of vertebral artery and middle cerebral artery monitoring for right-to-left shunt detection by contrast-enhanced transcranial Doppler. Scientific reports6, 1-6 (2016).

23 Kobayashi, K. et al. Right-to-Left-Shunt Detected by c-TCD Using the Orbital Window in Comparison with Temporal Bone Windows. Journal of Neuroimaging22, 80-84 (2012).

24 Trattnig, S., Schwaighofer, B., Hübsch, P., Schwarz, M. \& Kainberger, F. Color-coded Doppler sonography of vertebral arteries. Journal of ultrasound in medicine10, 221-226 (1991).

25 Chuang, Y.-M., Chan, L., Wu, H.-M., Lee, S.-P. \& Chu, Y.-T. The clinical relevance of vertebral artery hypoplasia. Acta Neurol Taiwan21, 1-7 (2012).

26 Agustin, S. J. U. et al. Effects of posture on right-to-left shunt detection by contrast transcranial doppler. Stroke42, 2201-2205 (2011).

$27 \mathrm{Wu}, \mathrm{C.}$-T. et al. Effects of patient position on right-to-left shunt detection by contrast transcranial Doppler. Ultrasound in medicine \& biology41, 654-658 (2015).

28 Topcuoglu, M. A. Diagnosis of right-to-left shunts by transcranial doppler in patients with insufficient temporal bone window. Stroke39, e123-e124 (2008).

29 Zarrinkoob, L. et al. Blood flow distribution in cerebral arteries. Journal of Cerebral Blood Flow \& Metabolism35, 648-654 (2015).

30 Furlan, A. J. et al. Closure or medical therapy for cryptogenic stroke with patent foramen ovale. New England Journal of Medicine366, 991-999 (2012).

31 Meier, B. et al. Percutaneous closure of patent foramen ovale in cryptogenic embolism. New England Journal of Medicine368, 1083-1091 (2013).

32 Carroll, J. D. et al. Closure of patent foramen ovale versus medical therapy after cryptogenic stroke. New England Journal of Medicine368, 1092-1100 (2013).

33 Jauss, M. \& Zanette, E. Detection of right-to-left shunt with ultrasound contrast agent and transcranial Doppler sonography. Cerebrovascular diseases10, 490-496 (2000).

34 Spencer, M. P. et al. Power m-mode transcranial Doppler for diagnosis of patent foramen ovale and assessing transcatheter closure. Journal of Neuroimaging14, 342-349 (2004). 

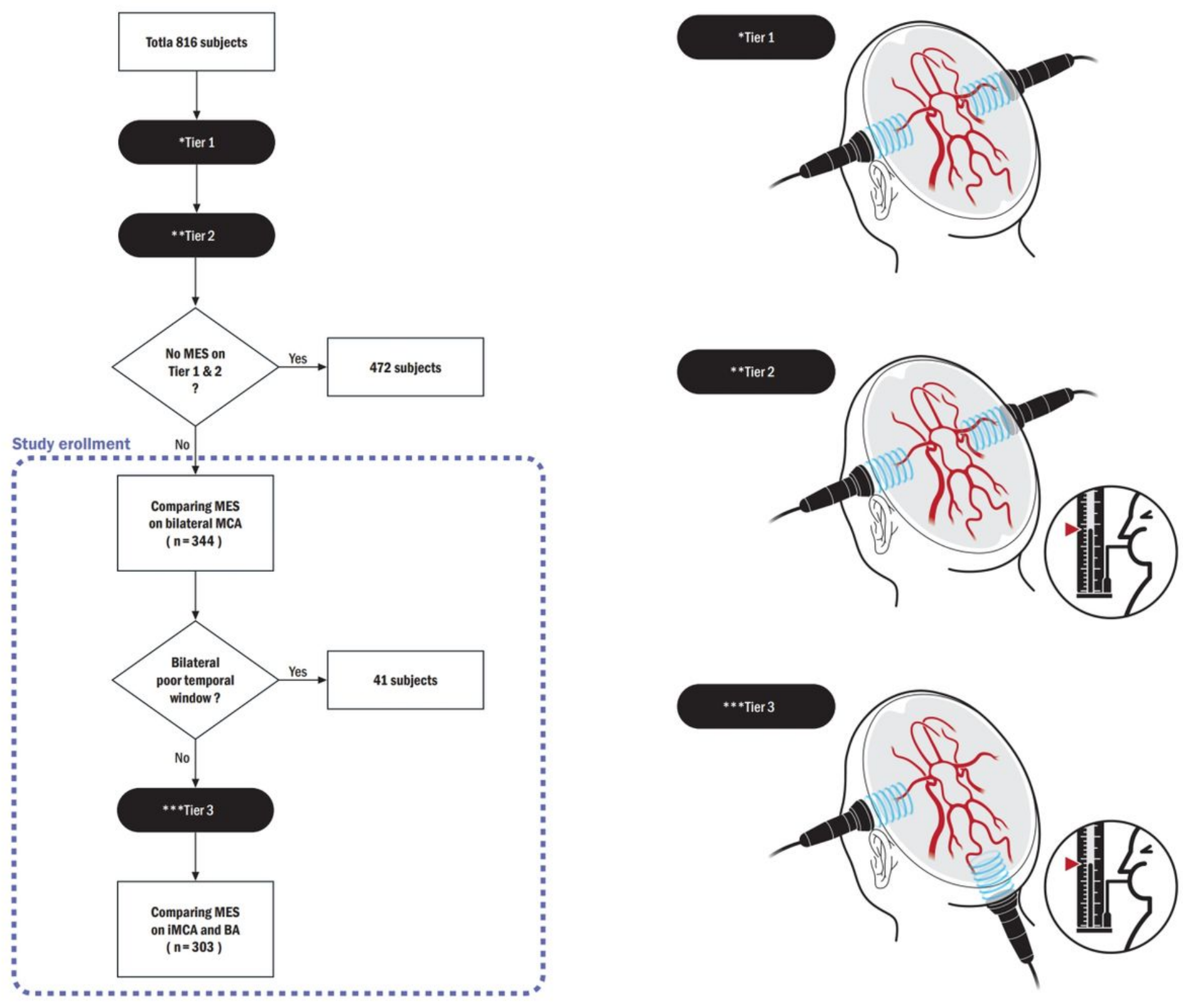

\section{Figure 1}

Flow diagram of study enrollment Tier 1*: Bilateral MCA (or ICA) monitoring on rest state Tier 2**: Bilateral MCA (or ICA) monitoring with Valsalva maneuver Tier $3 * \star *$ : Simultaneous monitoring of iMCA and BA with Valsalva maneuver MES: microembolic signals; PTW: poor temporal window; MCA: middle cerebral artery; BA: basilar artery; iMCA: index of MCA; ICA: internal carotid artery 


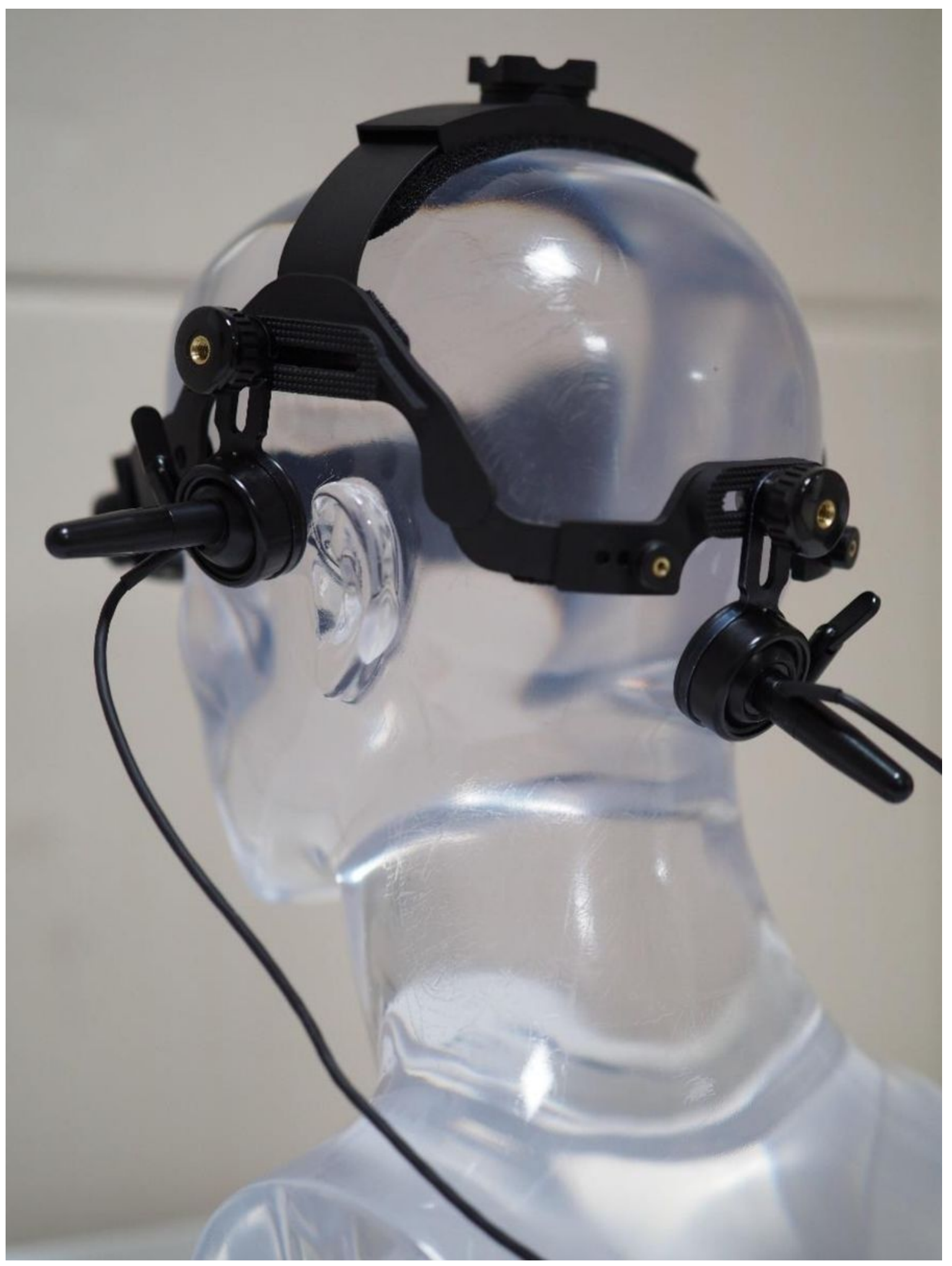

Figure 2

Fixable device which can be placed on the MCA and BA during TCD of the PFO 

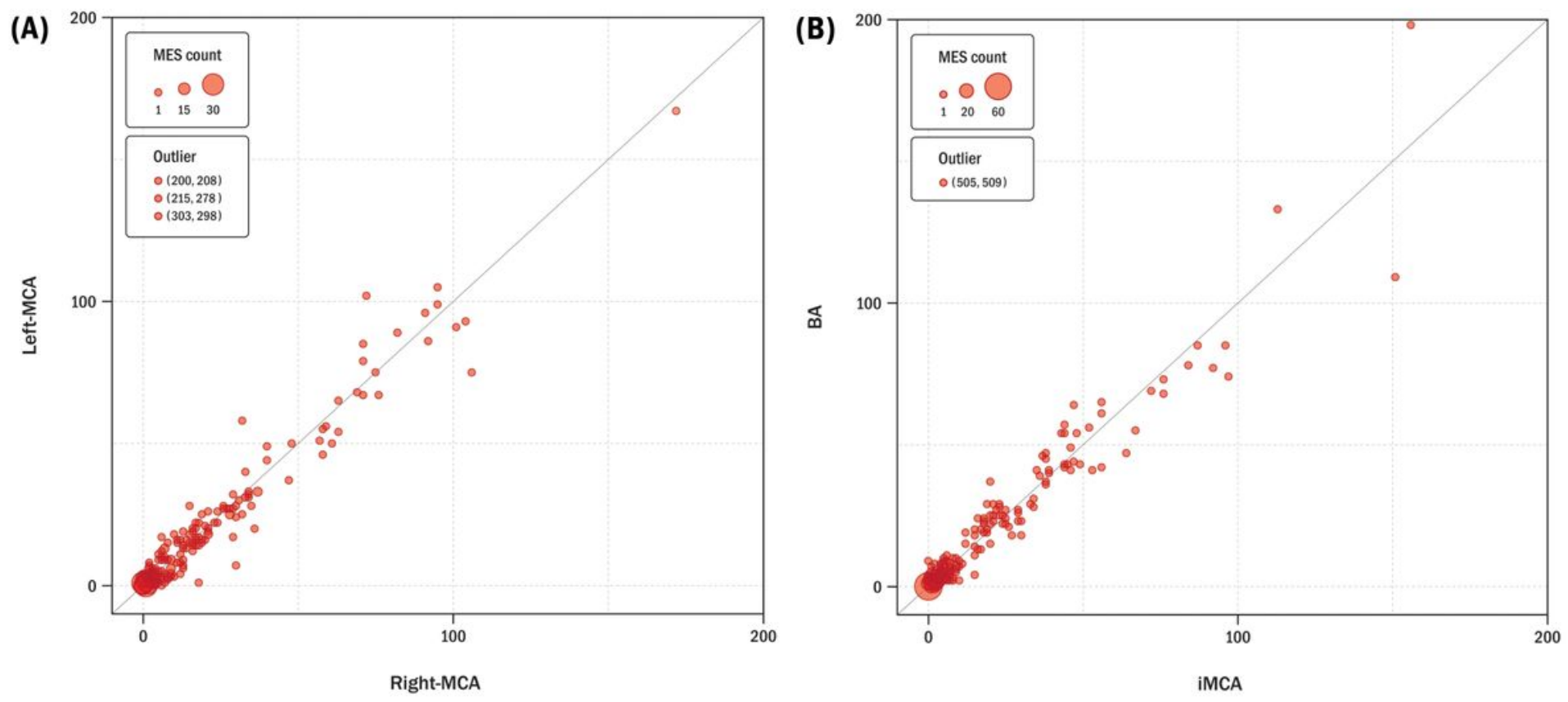

Figure 3

Comparison of TCD-PFO in the MCAs bilaterally or in the MCA and BA (A): The bubble counts in the MCAs bilaterally on TCD (Tier 2), ICC $=0.9822(p<0.001)(B)$ : The bubble counts in the iMCA and BA on TCD (Tier 3), ICC $=0.9860(p<0.001)$ 